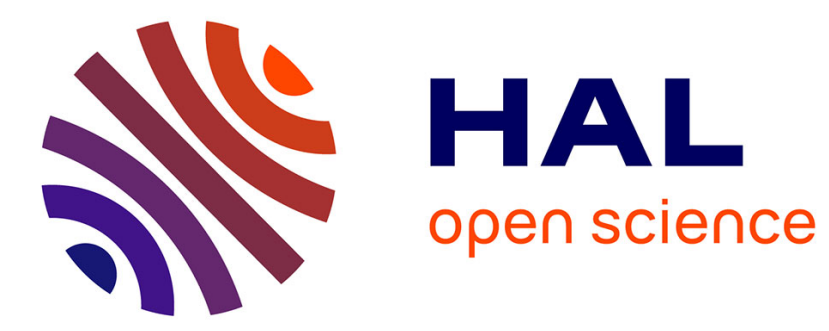

\title{
Mechanisms of Heparin-Induced Tau Aggregation Revealed by a Single Nanopore
}

Nicoletta Giamblanco, Yann Fichou, Jean-Marc Janot, Emmanuel Balanzat, Songi Han, Sebastien Balme

\section{- To cite this version:}

Nicoletta Giamblanco, Yann Fichou, Jean-Marc Janot, Emmanuel Balanzat, Songi Han, et al.. Mechanisms of Heparin-Induced Tau Aggregation Revealed by a Single Nanopore. ACS Sensors, 2020, 5 (4), pp.1158-1167. 10.1021/acssensors.0c00193 . hal-03035735

\section{HAL Id: hal-03035735 https://hal.science/hal-03035735}

Submitted on 7 Dec 2020

HAL is a multi-disciplinary open access archive for the deposit and dissemination of scientific research documents, whether they are published or not. The documents may come from teaching and research institutions in France or abroad, or from public or private research centers.
L'archive ouverte pluridisciplinaire HAL, est destinée au dépôt et à la diffusion de documents scientifiques de niveau recherche, publiés ou non, émanant des établissements d'enseignement et de recherche français ou étrangers, des laboratoires publics ou privés. 


\title{
Mechanisms of heparin-induced tau aggregation revealed by single nanopore
}

Nicoletta Giamblanco, ${ }^{\text {ta }}$ Yann Fichou, ${ }^{\text {fb }}$ Jean-Marc Janot, ${ }^{\mathrm{a}}$ Emmanuel Balanzat, ${ }^{\mathrm{c}}$ Songi Han, ${ }^{\mathrm{b}}$ and Sebastien Balme*a

anstitut Européen des Membranes, UMR5635 UM ENCSM CNRS, Place Eugène Bataillon, 34095 Montpellier cedex 5, France.

${ }^{b}$ Department of chemistry and biochemistry, University of California Santa Barbara, Santa Barbara, USA.

${ }^{C}$ Centre de recherche sur les lons, les Matériaux et la Photonique, UMR6252 CEA-CNRS-ENSICAEN, 6 Boulevard du Maréchal Juin, 14050 Caen Cedex 4, France.

¥ Both authors contributed equally to this manuscript.

*Correspondance : sebastien.balme@umontpellier.fr

\begin{abstract}
Protein aggregation is involved in many diseases, including Parkinson's and Alzheimer's ones. The latter is characterized by intraneuronal deposition of amyloid aggregates composed of the tau protein. Although large and insoluble aggregates are typically found in affected brains, intermediate soluble oligomers are thought to represent crucial species for toxicity and spreading. Nanopore sensors constitute an emerging technology that allows the detection of the size and populations of molecular assembly present in a sample. Here, we employed conical nanopores to obtain the particle distributions during tau aggregation. We identified three distinct populations, monomers, oligomers and fibrils, which we could quantify along the aggregation process. By comparing tau wild type with a mutant carrying the disease-associated P301L mutation, we showed that the latter mutation promotes the formation of oligomers. We furthermore highlighted that the P301L mutation promotes fibril breakage. This work demonstrates that conical nanopore is a powerful tool to measure and quantify transient protein aggregate intermediates.
\end{abstract}

Keyword : nanopore, amyloid, protein tau, P301L mutation, heparin-induced aggregation, kinetic, track-etched 
The tau protein is identified as a major component of proteinaceous deposition in abnormal brain characteristic of several neurodegenerative diseases termed tauopathies, including Alzheimer's disease ${ }^{1}$. Several studies have pointed out that prefibrillar soluble aggregates or oligomers are toxic species and that their presence in the brain correlates with cognitive deficits ${ }^{2-4}$. Despite the large body of evidence supporting the critical role played by tau oligomers in initiation and progression of the disease ${ }^{5}$, their structural and molecular identity are still scarcely described, in part due to a lack of suitable methods for their detection.

Tau is an intrinsically disordered protein (IDP) found in neurons where it regulates microtubules polymerization ${ }^{6,7}$. It is present in different isoforms that contain three or four repeat regions (R1 to R4), which form most of the core of the aggregate amyloid core ${ }^{8-10}$. Tau is highly hydrophilic and is therefore stable under physiological conditions and across a wide range of $\mathrm{pH}$ and temperatures. In vitro aggregation of tau is typically triggered by cofactors, most commonly heparin ${ }^{11}$, but also other cofactors such as RNA ${ }^{12}$ or arachidonic acid ${ }^{13}$. Although heparin-induced tau filaments were shown to be heterogeneous and different from existing brain-derived aggregates structures $^{14,15}$, it still represents a suitable model to study the mechanisms of tau amyloid filament formation.

Before forming stable amyloid fibrils, tau self assembles in heterogeneous and transient species with different structures, conformations and properties ${ }^{3,16-18}$. Individual tau mutations, which are involved in familial tauopathies and can promote in vitro aggregation ${ }^{19}$, promote aggregation and confer the ability to aggregate into conformationally distinct assemblies ${ }^{20}$. However, the detailed structural mechanisms by which disease-associated mutations promote aggregation is complex and not well understood. The ability to elucidate the kinetics of tau oligomerization harboring mutations is expected to provide new insights into the mechanisms modulating tau aggregation. There are numerous techniques to characterize protein aggregation ${ }^{21}$. The fluorescence of intercalant agents (such as thioflavine T) allows following the kinetic of fibrillation based on the presence of cross$\beta$ structures. Correlation methods (DLS and FCS) assess the size distributions ${ }^{22}$ but cannot easily provide information about the aggregate morphology. Super-resolution microscopy, (Cryo-)TEM, AFM and ssNMR allow to characterize the structure of amyloid fibrils in a near-atomic-resolution ${ }^{23}$. However, these characterizations are not suitable to follow the kinetic of amyloid growth." And P3 "The nanopore technology provides information about the aggregation kinetics as well as about the size of aggregates at a single molecule level, without the need of to add a label or a die.

To address this issue, the nanopore technology represents a promising approach ${ }^{24}$. The resistive pulse sensing method ${ }^{25}$ entails measuring current perturbation due to the presence of a single molecule inside the nanopore. The current perturbation is defined by a duration, an amplitude, a frequency and a shape that encode a signature or finger printing of the detected molecule ${ }^{26,27}$. In this field, the biological nanopores are the most extensively studied. They are sensitive, but have been limited to the characterization of very small peptides oligomers ${ }^{28,29}$. As an example, the $\alpha$-hemolysin was used to detect the aggregation of inhibition $A \beta(1-42)$. However, when the aggregates become too large, they do not translocate through the nanopore inducing only bumping events. In that case, it is impossible to obtain information about their size and their structures ${ }^{28}$. Solid-state nanopores are more suitable than the biological ones to detect protein aggregates, but they have to be functionalized since aggregation enhances the protein adsorption and thus their fouling ${ }^{30}$. Mayer's group has developed a new promising approach relying on SiN nanopores decorated with a lipid bilayer adsorbed on the SiN nanopore wall $^{31,32}$. Using this strategy, they successfully detected small amyloids of $\beta$-peptide. The functionalization of SiN by tween 20 were employed by Hu R. et al. ${ }^{33}$. The surfactant allows inhibiting non-specific adsorption and allows to oligomerization of $\alpha$-synuclein. Similarly, PEG-coated SiN nanopores have been shown to be suitable to discriminate between the morphology of BSA and lysozyme aggregates ${ }^{34}$. Quartz glass nanopore was also employed to characterize prion ${ }^{35}$,lysozyme ${ }^{36}$ and $\beta$-peptide ${ }^{37}$ aggregates without functionalization. However, the 
main limitations of the aforementioned technologies are (i) their sizes that do not allow the detection of large assemblies, (ii) their short lifetime and (iii) the difficulties to be functionalized. To tackle these bottlenecks, we recently reported that conical nanopore in polyethylene terephthalate (PET) obtained by track-etched methods and pore-functionalized with polyethylene glycol $5 \mathrm{kDa}\left(\mathrm{PEG}_{5 \mathrm{k}}\right)$ is likely the most suitable candidate to investigate the early stage oligomerization ${ }^{38}$. Indeed, this new design was shown to be capable of following the amyloid growth of $\beta$-lactoglobulin while discriminating between oligomers and fibrils population ${ }^{38}$. Compared to the conventional technique, the nanopore can provide under continuous measurement the information about the kinetic aggregation given in at the same time information about the size of aggregate at single molecule level.

Following this proof of concept, the present study aims to characterize the transient species formed during the heparin-induced aggregation of tau with and without a disease mutation using conical track-etched nanopore in PET. In particular, we have investigated tau187, a truncated version of the longest human tau protein isoform (residues 255 to 441 ), in the absence and presence of the disease mutation $\mathrm{P}^{2} 01 \mathrm{~L}^{39}$. This truncation includes all four microtubule-binding repeat domains as well as the C-terminal region. We followed the kinetic of different populations of tau aggregation using the single conical nanopore technology. We could extract from the signal numerous information about the aggregates such as the relative size and conformational flexibility ${ }^{40}$.

\section{Materials and methods}

\section{Materials}

Polydisperse heparin (15 kDa) was purchased from Galen Laboratory Supplies (HEP001). . AminoPEG 5 kDa $\left(\mathrm{PEG}_{5 \mathrm{k}}-\mathrm{NH}_{2}\right)$ was purchased from Nanocs (PG1-AM-5K). 1-ethyl-3-(-3-dimethylaminopropyl) carbodiimide hydrochloride (EDC, E7750), MES hydrate (M8250), ammonium acetate $\left(\mathrm{NH}_{4} \mathrm{AC}\right)$, potassium chloride ( $\left.\mathrm{KCl}, \mathrm{P3911}\right)$, sodium hydroxide ( $\mathrm{NaOH}, \mathrm{S5881})$ and sodium chloride $(\mathrm{NaCl}, 71380)$, were purchased from Sigma-Aldrich. Minisart syringe filters (16553-K) $0.1 \mu \mathrm{m}$ were purchased from Sartorius stedim.

\section{Protein production}

Purified DNA consisting of residues 255-441 of tau isoform 2N4R was used to express tau187. Site-directed mutagenesis was used to introduce the $\mathrm{P} 301 \mathrm{~L}$ mutation into the tau187 plasmid. . The success of the mutagenesis was ensured by Sanger sequencing, performed at Genewiz. The proteins were expressed in E. coli BL21 (DE3), followed by purification steps as described elsewhere ${ }^{41}$. Briefly, BL21 (DE3) cells transfected with a pET28a vector including the tau187 genes with and without P301L were grown in $1 \mathrm{~L}$ cultures of LB broth (Fisher Scientific) until an optical density of 0.6-0.8 at $600 \mathrm{~nm}$ was achieved. Then expression was induced through addition of $1 \mathrm{mM}$ isopropyl $\beta$-D-1-thiogalactopyranoside (IPTG, Fisher Scientific) and incubated for $3 \mathrm{~h}$. Cells were harvested by centrifugation at $6000 \mathrm{xg}$ (Beckman J-10) for $20 \mathrm{~min}$. Purification was carried out by lysing the cells with $2 \mathrm{mg} \cdot \mathrm{mL}^{-}$ ${ }^{1}$ lysozyme (Sigma Aldrich) while performing 3 cycles of freeze fracture in liquid nitrogen, before pelleting cell debris through centrifugation. Subsequently, the supernatant was heated to $65^{\circ} \mathrm{C}$ for $12 \mathrm{~min}$ and precipitated proteins were removed by centrifugation. Tau was extracted from the supernatant by binding to nickel affinity resin and subsequently removed by washing with increasing concentration of imidazole buffers. . For each batch, the protein purity was verified to be above $\sim 80 \%$ by performing SDS-page electrophoresis.

\section{Heparin induced tau187WT and tau187P301L fibrillation}

Tau187WT and Tau187P301L (19.6 $\mu \mathrm{M})$ and heparin $(5 \mu \mathrm{M})$ in $20 \mathrm{mM} \mathrm{NH}_{4} \mathrm{Ac}, 100 \mathrm{mM} \mathrm{NaCl} \mathrm{pH} 7.2$ were prepared as a stock solution. The samples were filtered $(0.1 \mu \mathrm{m})$ before used. The fibrillation of tau187WT and 
tau187P301L was performed at $25{ }^{\circ} \mathrm{C}$ by addition of a protein solution $(9.8 \mu \mathrm{M})$ with heparin $(2.6 \mu \mathrm{M})$ corresponding to a tau: heparin molar ratio of $3.8: 1$. For each time point, aliquots were taken.

\section{Thioflavin T fluorescence experiment.}

Protein aggregation was monitored by Thioflavin T (ThT) fluorescence using $20 \mu \mathrm{M}$ ThT in a plate reader Biotek synergy 2. Both tau187P301L and tau187WT were measured in the same plate at a concentration of $10 \mu \mathrm{M}$ after addition of $2.5 \mu \mathrm{M}$ heparin. Colored area around the curves represents standard deviation over three repeats.

\section{Transmission electron microscopy}

Tau187WT and P301L were incubated with heparin for 24h. A TEM grid, FORMVAR Carbon film on 300 meshes copper (Electron Microscopy Sciences), was floated on $10 \mu \mathrm{L}$ of the sample for $1 \mathrm{~min}$ and then blotted on filter paper. The grid was touched to $10 \mu \mathrm{L}$ of deionized water, blotted, touched to $10 \mu \mathrm{L}$ of the stain solution, $2 \%$ Uranyl Acetate (Electron Microscopy Sciences), blotted and then floated on $10 \mu \mathrm{L}$ of $2 \%$ Uranyl Acetate for 1 minute before blotting and setting aside to dry for $24 \mathrm{~h}$. Imaging was carried out at room temperature using a JEOL JEM-1230 TEM.

\section{Fabrication and caracterisation of Single Conical Nanopore by the Track-Etching Technique.}

Single tracks were produced by Xe irradiation ( $\left.8.98 \mathrm{MeV} \mathrm{u}^{-1}\right)$ of PET film $(6 \mu \mathrm{m})$ (GANIL, SME line, Caen, France). The activation of the track was performed by UV exposition for $8 \mathrm{~h}$ for the tip side and $11 \mathrm{~h}$ for the base side, (Fisher bioblock; VL215.MC, $\lambda=312 \mathrm{~nm}$ ) before the chemical etching process. The etching of the conical nanopore was performed under dissymmetric conditions as previously reported ${ }^{42,43}$. After the nanopore etching, the film was stabilized over-night in $18 \mathrm{M} \Omega$ water. The approximate tip diameter was obtained by conductance measurement. After the chemical etching, the current-voltage (I-V) curve was measured at $\mathrm{pH} 5.6$ (black square, Figure S-1). Then the nanopore was functionalized with $\mathrm{PEG}_{5 \mathrm{k}}-\mathrm{NH}_{2}$ using 1-ethyl-3-(-3-dimethylaminopropyl) carbodiimide hydrochloride as previously reported in order to prevent the unspecific adsorption and improve the nanopore lifetime ${ }^{38}$. The success of the grafting was confirmed by the decrease of I-V curve slope due to the replacement of COO- by the PEG which diminished diameter of the nanopore.

\section{Nanopore sensing of tau187WT and tau187P301L}

The nanopore functionalized with PEG moieties was mounted between two compartments of a Teflon cells filled with buffered $1 \mathrm{M} \mathrm{KCl}$ aqueous solution $(\mathrm{pH}=5.6)$. The tau187WT or tau187P301L at different aggregation times (from 0 to $376 \mathrm{~min}$ ) was added to the base side to reach a concentration of $8.2 \mathrm{nM}$ (monomer equivalent). The resistive pulse experiments were performed using a patch-clamp amplifier (EPC 10, HEKA electronics, Germany) with $\mathrm{Ag} / \mathrm{AgCl}$ electrodes. A voltage of $-200 \mathrm{mV}$ was applied to the working electrode located in the tip side compartment to drive the positively charged protein tau, (isoelectric point of $8.24^{44}$ ) to pass through the nanopore from the base to tip side. The ionic currents were recorded at $20 \mathrm{kHz}$. The signal filtered at $2.9 \mathrm{kHz}$ by a Bessel filter. The resistive pulse detection was recorded on fly during 1 minute at least 10 times.The current traces were further analyzed to detect events using lab-made software "Peak Nano Tools" developed using Labview. First the signal was filtered using Bessel filter $1 \mathrm{kHz}$. The threshold for the event detection was defined as follow: (i) correct the baseline using Stavinsky-Golay filter (ii), define the noise levels by the global standard deviation methods (iii) define the threshold. In this work, the threshold has been fixed at about $5 \sigma$.

\section{Results and discussion}




\section{Detection of tau187WT and tau187P301L monomer and aggregates using conical nanopores}

The characterization of heparin-induced aggregates of truncated variant tau187 without (tau187WT) and with the P301L mutation (tau187P301L ; figure 1a) was performed through a single nanopore using the resistive pulse method $^{24}$. We used conical nanopores in PET obtained by track-etched methods ${ }^{42}$ and pore-functionalized with $\mathrm{PEG}_{5 \mathrm{k}}$ chains since it prevents the protein adsorption, and thus ensures a long lifetime of the nanopore ${ }^{38,45}$ (Figure $\mathrm{SI}-1)$. Tau aggregation was induced by mixing tau187 with hepari ${ }^{11}$ and was followed by thioflavin T fluorescence (ThT) (Figure 1b). ThT characterizes the cross $\beta$-sheet structure formation but does not provide information of the different species in the mixture. The formation of fibrillary assemblies was confirmed by transmission electron microscopy (Figure 1c, d). We measured the protein samples by nanopore at various incubation times from $0 \mathrm{~min}$ to $372 \mathrm{~min}$, as indicated in figure $1 \mathrm{~b}$, during the fibrillation process.
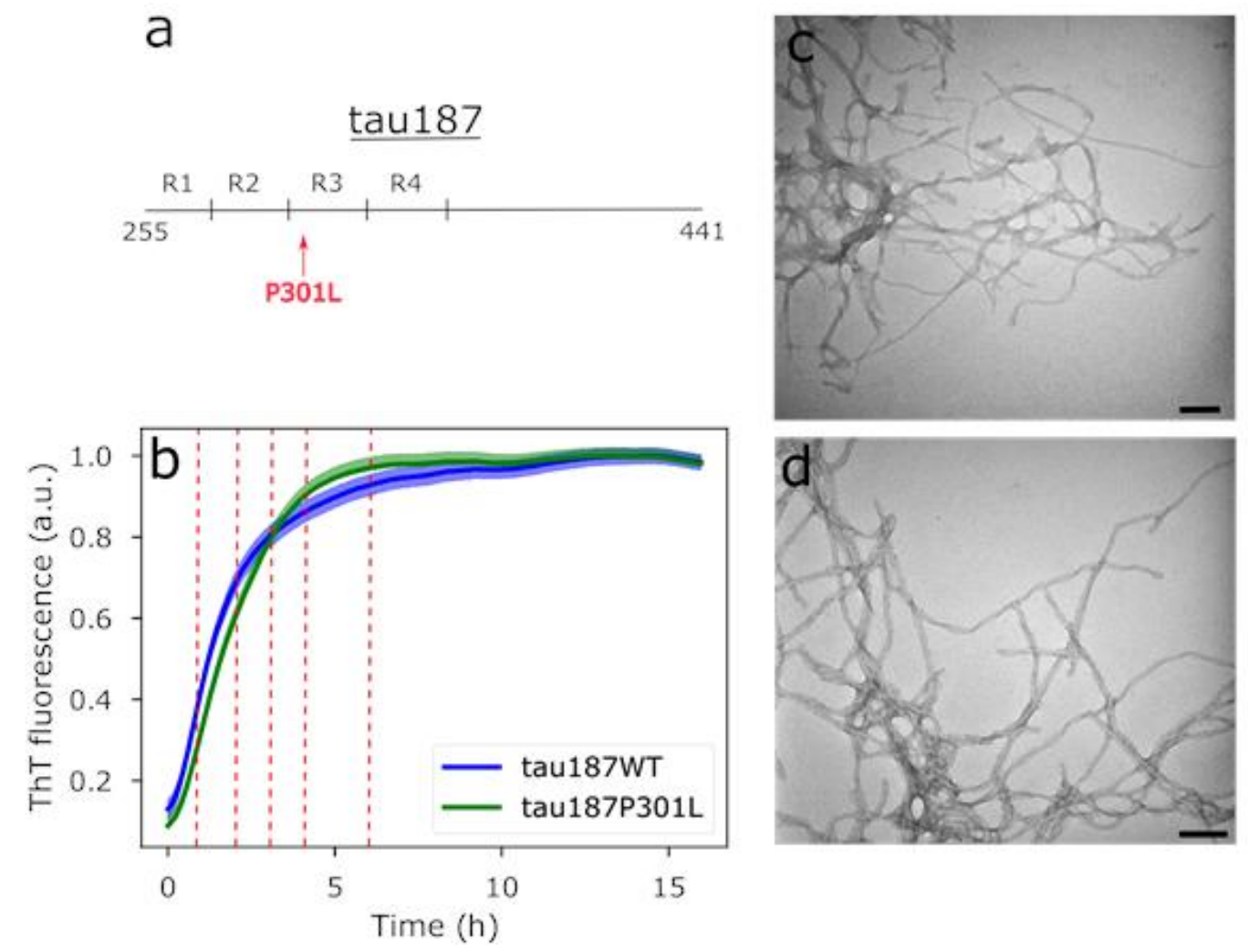

Figure 1 a) Tau187 consists of four repeat domains indicated as R1-R4. The position at the mutated proline P301L is indicated in red. b) ThT fluorescence curves of heparin-induced aggregation for tau187WT and tau187P301L. The red dash lines represent the approximate times at which aliquots were extracted for nanopore measurements. The faint areas around the curves represent the standard deviation over 3 replicates of the aggregation reaction. The formation of fibrils $24 \mathrm{~h}$ after incubation with heparin was confirmed by TEM for tau187WT (c) and tau187P301L (d). The scale bar is $100 \mathrm{~nm}$.

The heparin-induced aggregates were detected from the base to the tip side of the nanopore under applied voltage $-200 \mathrm{mV}$ at $1 \mathrm{M} \mathrm{KCl}$ and pH 5.6 (Figure 2a). Several examples of current traces obtained for Tau187WT and tau187P301L before and after heparin addition are reported on figure $2 \mathrm{~b}-\mathrm{g}$ and $2 \mathrm{~h}-\mathrm{m}$ respectively. The passage of an object inside the detection zone induces a current blockade characterized by the amplitude $\left(\Delta l / I_{0}\right)$ and the dwell time $(\Delta t)$, as previously described ${ }^{38}$. 

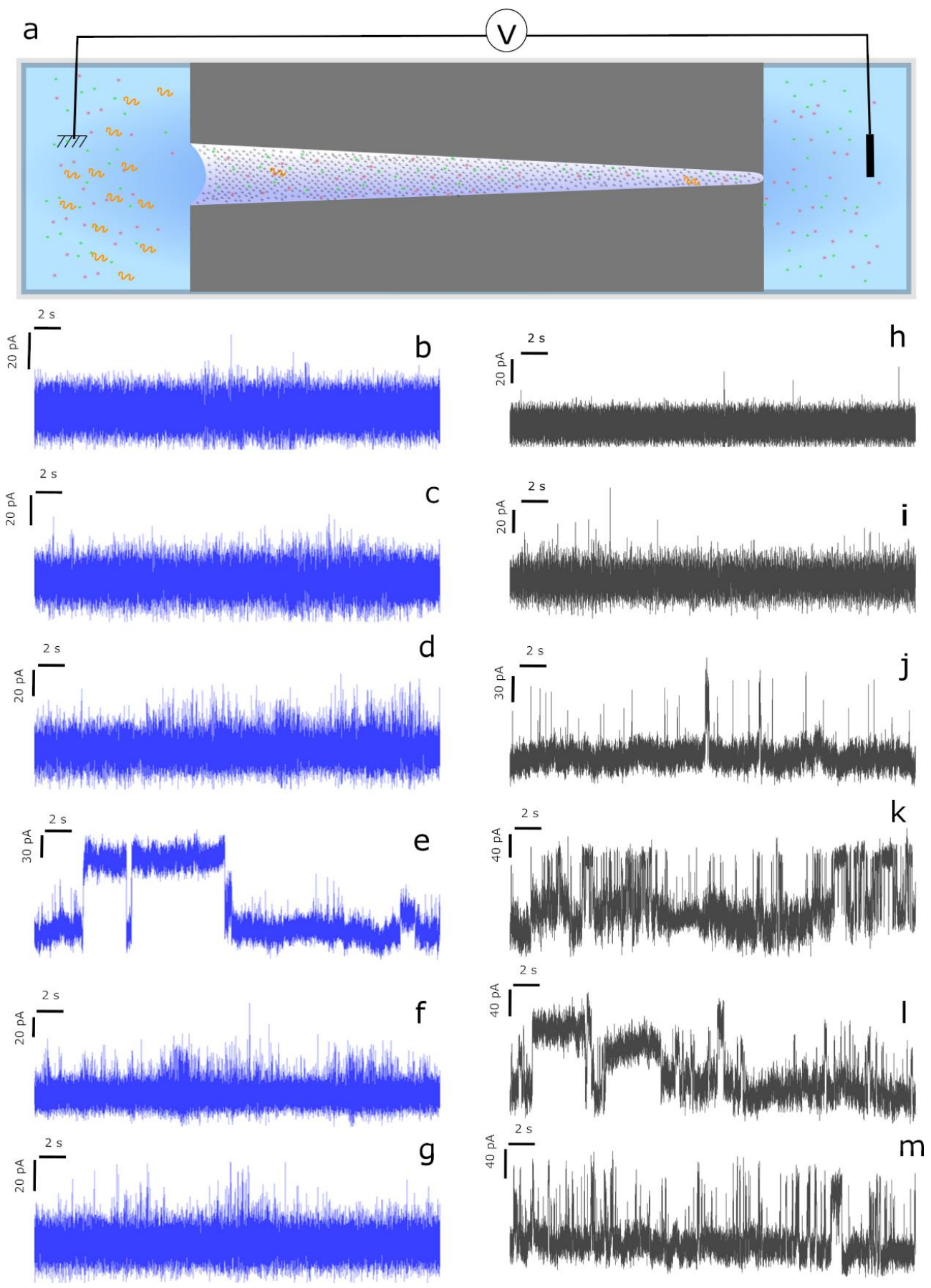

Figure 2. Electric field-driven translocation of tau protein. (a) Sketch of resistive pulse experiments: the positively charged tau is driven through the nanopore by the applied negative voltage $(-200 \mathrm{mV})$. Current traces obtained for tau187WT before heparin addition (b) and after $70 \mathrm{~min}$ (c), $130 \mathrm{~min}$ (d), $198 \mathrm{~min}(\mathrm{e}), 256 \mathrm{~min}$ (f) and $372 \mathrm{~min}$ (g) and for tau187P301L before heparin addition (h) and after $60 \mathrm{~min}$ (i), $140 \mathrm{~min}(\mathrm{j}), 198 \mathrm{~min}(\mathrm{k}), 260 \mathrm{~min}(\mathrm{I})$ and $346 \mathrm{~min}(\mathrm{~m})$. These traces were obtained for a protein concentration (C= 8.2 $\mathrm{nM}$ ) under $1 \mathrm{M}$ $\mathrm{KCl} \mathrm{pH} 5.6$ at $-200 \mathrm{mV}$ through conical nanopore $r_{\text {base }}=90 \mathrm{~nm}$ and $r_{\text {tip }}=6 \mathrm{~nm}, \mathrm{~L}=6 \mu \mathrm{m}$ 

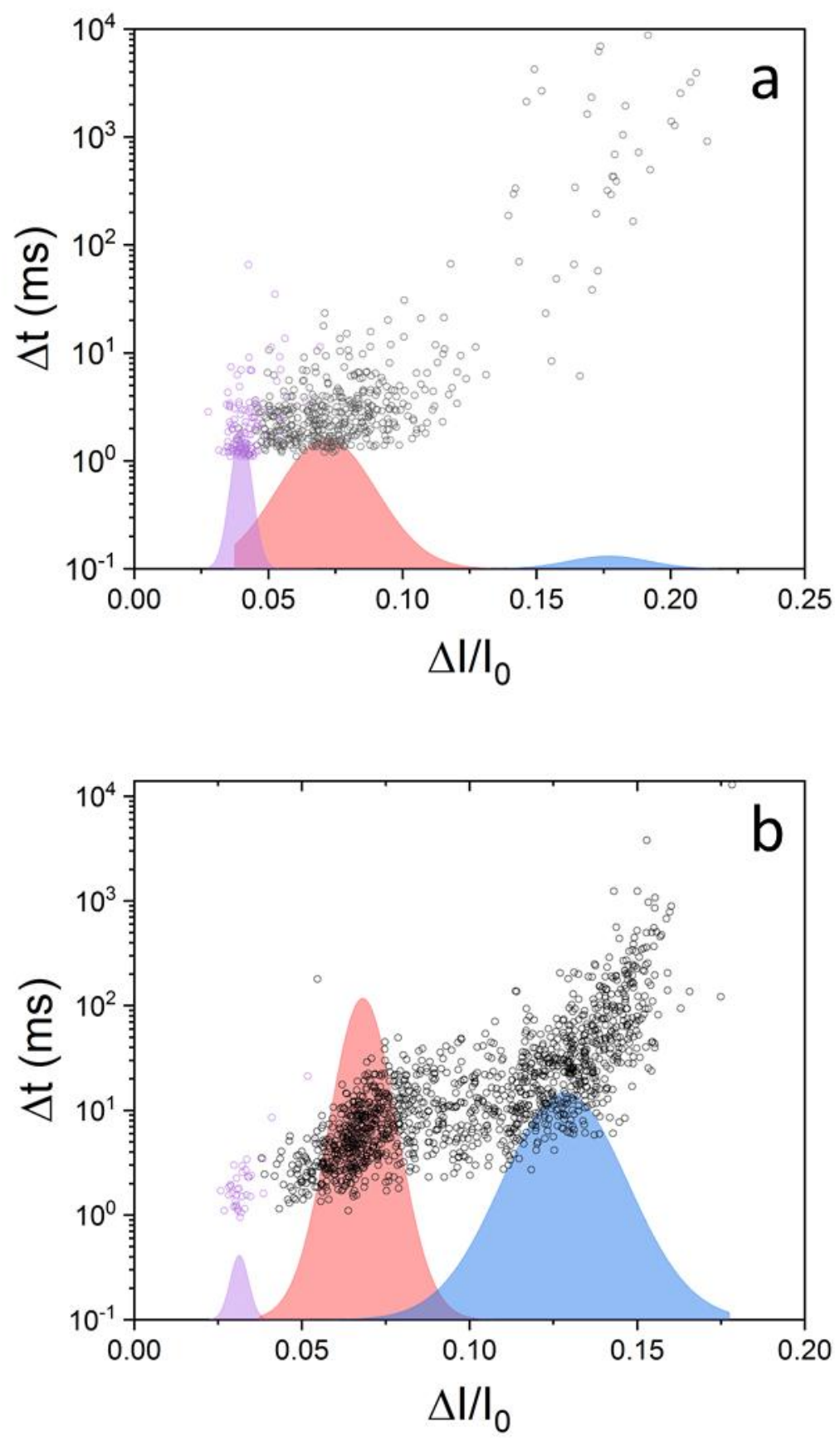

Figure 3. Scatter plot of translocation events for tau187WT (a) and tau187P301L (b) before (violet circles) and after $198 \mathrm{~min}$ heparin incubation (black circle). The reported Gaussian areas are obtained from a fit of the populations as reported in figure 4 (figure $4 \mathrm{a}$ and $4 \mathrm{~d}$ for tau187WT and figure $4 \mathrm{~g}$ and $4 \mathrm{j}$ for tau187P301L).

We report the 2D event maps (Figure 3, SI-2 and SI-3) that characterize how the $\Delta t$ vs $\Delta \mathrm{I} / \mathrm{I}_{0}$ of tau187WT and tau187P301L vary between before and after heparin addition. The dwell time $(\Delta t)$ slightly increases with $\Delta l / I_{0}$ until a value about 0.12 . Then, when the $\Delta I / I_{0}$ is above $\sim 0.12$, the events become significantly longer. This means that there is not a simple dependence between the aggregate size and their dwell time inside the nanopore as shown for the translocation of macromolecule through biological nanopore ${ }^{27,46}$. This experimental result, which might seem counter intuitive, was previously reported experimentally for $\beta$-lactoglobulin and explained by modelling ${ }^{38}$. It was shown that with the increase of aggregate length the electric force and the hydrodynamic drag acting on 
the aggregates have opposite effects. The first one induces a decrease of dwell time whereas the hydrodynamic drag increases the dwell time. The event maps reported for tau187WT and tau187P301L (Figure 3a and 3b respectively) allow us to distinguish between the different populations of aggregates. The monomers of tau187WT and tau187P301L (violet Gaussian) population are characterized by $\Delta l / I_{0}<0.045$ and $\Delta t<10^{1} \mathrm{~ms}$. After heparin addition, populations of events possessing different $\Delta I / I_{0}$ can be discriminated, which can be assigned to different sizes of aggregate (see next section). From the events map, we can therefore distinguish whether the events belong to tau187 monomers or higher order assemblies. This is consistent with a previous study reporting the coexistence of different aggregates ${ }^{18,47,48}$.

We analyze next the distribution of the relative current blockade before heparin addition (corresponding to monomers) and after various incubation times. Before heparin addition, the $\Delta l / l_{0}$ distribution obtained for tau187WT is narrow and fits well to a monomodal Gaussian distribution centered to 0.039 for tau187WT (figure 4a). After $70 \mathrm{~min}$ of incubation, the distribution is still monomodal centered on 0.046 , suggesting that oligomerization has occurred (figure $4 \mathrm{~b}$ ). Although we observed an overlap between the $\Delta l / l_{0}$ distributions of monomer and after $70 \mathrm{~min}$ of incubation (figures $4 \mathrm{a}$ and $4 \mathrm{~b}$ respectively), the distribution centers are clearly distinct, indicating that a part of the monomers are oligomerized after $70 \mathrm{~min}$, in agreement with previous reports ${ }^{16}$. Attempts to fit the distribution observed at $70 \mathrm{~min}$ with two Gaussians (intended to represent the monomeric and oligomerized populations) failed given the narrow distribution and the low resolution of the nanopores for small objects. After $130 \mathrm{~min}$ of incubation (figure 4c), the $\Delta l / I_{0}$ distribution requires two components centered on 0.040 and 0.057 , revealing the presence of two distinct populations of tau187WT. The first $\Delta l / I_{0}$ component (violet, figure $4 \mathrm{c}$ ) represents the monomer population, while another component reflect an additional population representing oligomers (red, figure 4c). After $198 \mathrm{~min}$ of incubation (Figure 4d), the $\Delta l / I_{0}$ distribution requires also two Gaussian components centered respectively on 0.071 and 0.17 that can be assigned to different aggregate populations, referred to as oligomers and fibrils. The first distribution does not overlap with the monomer one (figure 4a) showing that the monomer population is completely converted to aggregates. The $\Delta \mathrm{l} / \mathrm{I}_{0}$ distributions are also broad indicating that heterogeneous aggregates were passing through the nanopore. After $256 \mathrm{~min}$ (figure 4e), the majority the two distributions are centered about 0.11 and 0.16 , where the oligomer population (red, fig 4e) is growing. Interestingly, after $372 \mathrm{~min}$ (figure $4 \mathrm{f}$ ), $\Delta l / \mathrm{I}_{0}$ distributions reveals one population centered to 0.064 , suggesting a resolution artefact and/or a fibril breakage (see discussion below).

For the mutant tau187P301L (figure 4g-k), the heparin-induced aggregation also reveals several populations that however follow distinct kinetic compared to tau187WT. Before heparin addition (Figure 4g), the relative current blockade distribution is monomodal centered to 0.031 , close the one obtained for tau187WT (0.039). After 60 min (figure 4h), one populations centered around 0.041 cannot be distinguished from the monomer (Figure $3 \mathrm{~g}$ ). After $130 \mathrm{~min}$ incubation time (figure 4i), two populations are detected. In contrast with tau187WT, the $\Delta l / I_{0}$ distribution does not overlap at all with the monomer distribution (figure $3 \mathrm{~g}$ ), showing that all monomers are converted at $130 \mathrm{~min}$. This confirms a faster formation of large oligomers for the mutant P301L than for WT. For the longer time, $198 \mathrm{~min}$ (figure $4 \mathrm{j}$ ) to $346 \mathrm{~min}$ (figure $4 \mathrm{l}$ ), the $\Delta l / I_{0}$ distributions remain bimodal, with a fibril population (bleu Gaussian) fitted around 0.2. The centers of $\Delta l / I_{0}$ distributions remain similar, about 0.13 and 0.2 . 

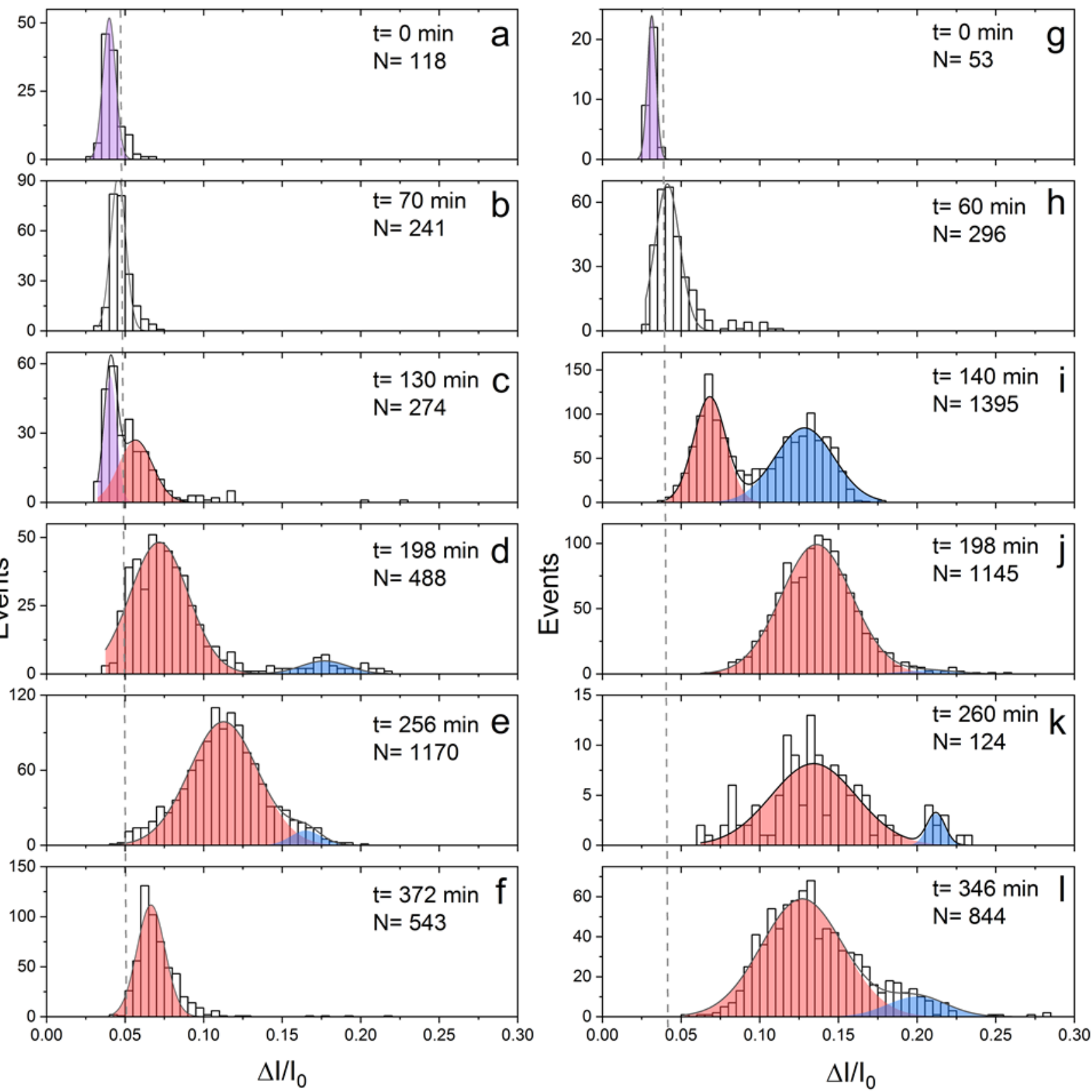

Figure 4. Nanopore-based analysis of tau187WT (a-f) and tau187P301L (g-l). Relative current blockade $\left(\Delta I / I_{0}\right)$ histograms of translocation of before $(\mathrm{t}=0 \mathrm{~min})$ and after various incubation time with heparin at $25^{\circ} \mathrm{C}$. The applied voltage is $-200 \mathrm{mV}$. The histograms were fitted by multi-Gaussian function. The vertical dash line correspond to the end of $\left(\Delta l / I_{0}\right)$ distribution of monomer, in order to guide eyes. $N$ corresponds to the ne number of events.

The apparent reduction for tau187WT at longer time (figure 4f) of aggregate size might originate from two phenomena, (i) fibril breakage occurring in the sample ${ }^{49}$ and (ii) the impossibility to detect large fibrils. The effect (ii) is detailed as follow. The detection is triggered by the entrance of aggregate inside the nanopore. As previously demonstrated, the capture rate $(f)$ is mainly due to the diffusion process (equation 1$)^{30,50,51}$.

$f=2 \pi D c R_{c}$

where $D$ is the diffusion coefficient of the aggregate, $c$ the concentration and $R_{c}$ the capture radius that is a little bit bigger than the nanopore radius. So the aggregate growth involves (i) a decrease of the object concentration due to the consumption of monomer and oligomer and (ii) a decrease of the diffusion coefficient. In this case, the 
probability of a large fibril to enter inside the nanopore becomes lower that the acquisition time. Additionally, to enter inside nanopore, the aggregates have to overcome an energy barrier that is not negligible as soon as the amyloid fibril size becomes close to the nanopore base diameter (about $230 \mathrm{~nm}$ ). Given that the fully grown tau fibrils are on the order of a $\mu \mathrm{m}^{49}$, we expect the large fibrils to be almost invisible to our technique. This is corroborated by the decrease of frequency of event at $372 \mathrm{~min}$ (Figure SI-4).

We observed that at long time tau187P301L (figure 4l) exhibits larger populations than tau187WT (figure 4i). We interpret this result by an enhanced fragmentation of P301L fibrils, compare to WT fibrils, that converts large fibrils (undetected) into the detectable oligomers/aggregates. This finding is in good agreement with previous single-molecule fluorescence study ${ }^{49}$ show more fragmentation for tau P301L. We also observe that the frequency of event obtained for P301L increases with the heparin incubation time (Figure SI-4) conversely to the WT. In addition, at long time, the frequencies of events are higher for tau187P301L than tau187WT (2.8 events s $\mathrm{s}^{-1}$ and 0.64 events $\mathrm{s}^{-1}$ respectively). In other words, we detect more events for P301L compare to WT, confirming that P301L fibrils fragment into detectable aggregates, as opposed to WT where less detectable aggregates (lower effective concentration) are present.

\section{Kinetic of tau187WT and tau187P301L fibrillation}

The kinetics of each of the two populations identified in figure 4, referred to as oligomers (red) and fibrils (blue), were analyzed. We report in figure 5 the time evolution of the mean value of each $\Delta l / I_{0}$ (which report on the size of the aggregates) for tau187WT and tau187P301L.

In both cases, the populations of aggregates could be fitted with a sigmoidal growth, which is typical of protein amyloid aggregation ${ }^{52-57}$ (equation 2).

$\frac{\Delta I}{I_{0}}=\left(\frac{\Delta I}{I_{0}}\right)_{i}+\frac{\left(\frac{\Delta I}{I_{0}}\right)_{f}-\left(\frac{\Delta I}{I_{0}}\right)_{i}}{1+\exp \left(-k\left(t-t_{1 / 2}\right)\right)}$

where $\left(\Delta I / I_{0}\right)_{f}$ is the final value for the observed population, $t_{1 / 2}$ is the midpoint and $k$ is an apparent growth rate (noted $k_{o}$ and $k_{f}$ for oligomers and fibrils respectively). 

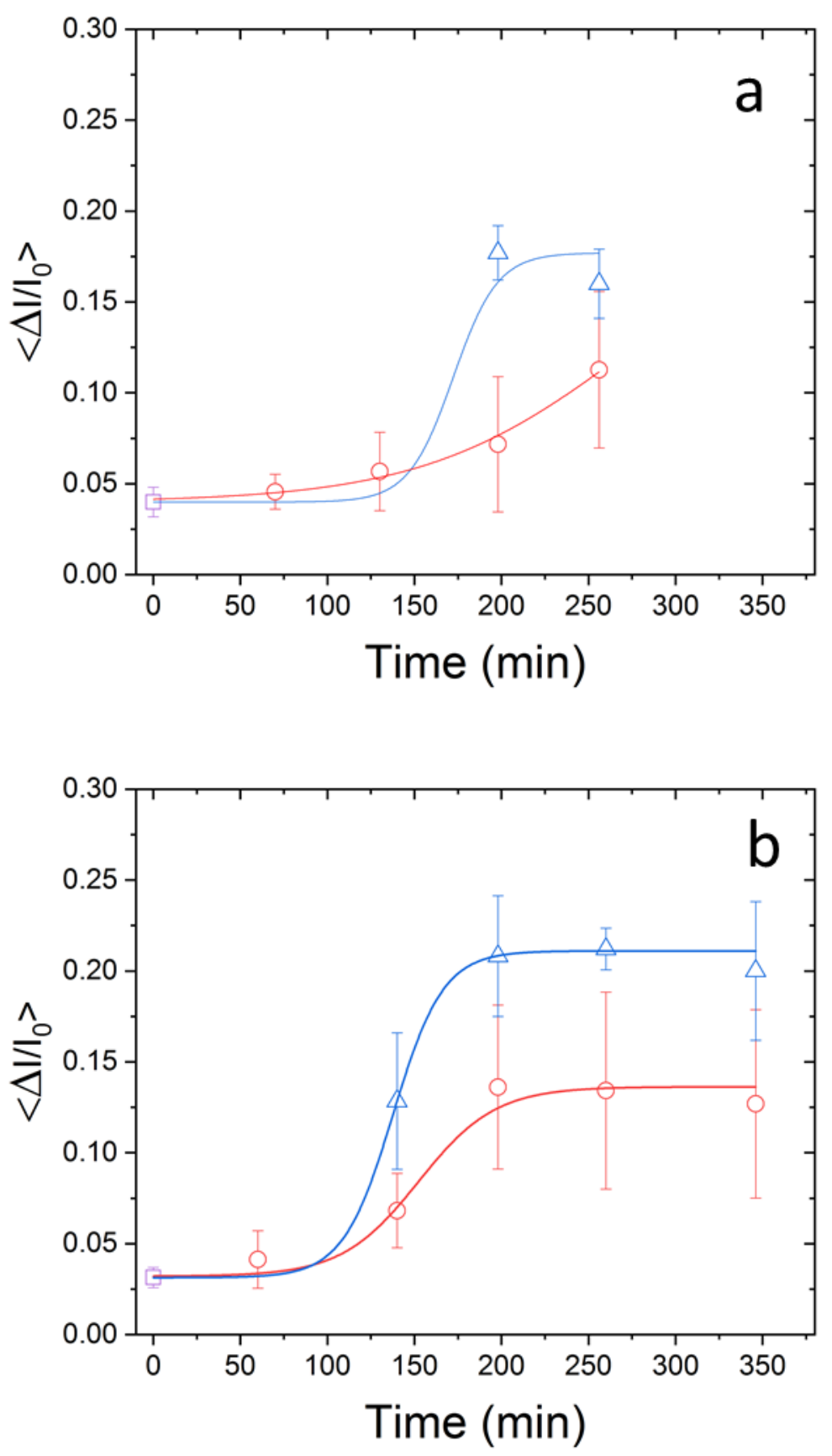

Figure 5. Kinetic of tau aggregation induced by heparin. Mean values of relative current blockade $\left(\Delta l / I_{0}\right)$ for the oligomers (red, circle), and fibrils (blue, triangle) versus the time of incubation with heparin for tau187WT (a) and tau187P301L (b). The error bars are the full width at half maximum of Gaussian distributions. The lines represent the fits of the different population using equation 2.

The result of the experimental data fitted by equation 2 are reported Table 1 . The apparent elongation rate of tau187WT fibrils $\left(k_{f}=7.810^{-2} \mathrm{~min}^{-1}\right)$ is similar to the one reported, based on absorption, for the tau 244-372 fragment (for molar ratio tau/heparin of $4, k=1010^{-2} \mathrm{~min}^{-1}$ ) 58 . The elongation rate constant of oligomers are faster for tau187P301L $\left(k_{o}=4.510^{-2} \mathrm{~min}^{-1}\right)$ than for tau187WT $\left(k_{o}=1.710^{-2} \mathrm{~min}^{-1}\right)$. In contrast, the formation rate constants of fibrils $k_{f}$ are higher for tau187WT than for tau187P301L $\left(k_{f}=7.810^{-2} \mathrm{~min}^{-1}\right.$ and $k_{p}=6.910^{-2} \mathrm{~min}^{-1}$ respectively), although the difference is rather small. We can therefore see that the P301L mutation has its most prominent effects on efficiently converting monomers to oligomers. This explains why after 130 min there is no 
detectable monomer population of tau187P301L (figure 4i), while it takes 198min for tau187WT (figure 4d). This result is consistent with the previous report by Kundel et $a l^{49}$ and Shammas et $a l^{18}$ showing that P301 mutation induce a faster fibril growth compared to the WT.

Table 1. Kinetic parameters extracted from fitting the relative current blockade versus time by using equation $\mathbf{2}$ and $3 . \boldsymbol{k}_{o}$ and $\boldsymbol{k}_{f}$ are the apparent growth rate of oligomers and fibrils, respectively and $t_{1 / 2}$ is the midpoint.

\begin{tabular}{|l|l|l|l|}
\hline & & tau187WT & tau187P301 \\
\hline Oligomers & $t_{1 / 2}(\mathrm{~min})$ & 271 & 152 \\
\hline & $k_{o}(\min -1)$ & $1.710^{-2}$ & $4.510^{-2}$ \\
\hline Fibrils & $t_{1 / 2}(\mathrm{~min})$ & 172 & 138 \\
\hline & $K_{f}\left(\mathrm{~min}^{-1}\right)$ & $7.810^{-2}$ & $6.910^{-2}$ \\
\hline
\end{tabular}

To further investigate the mechanisms of both tau187WT and tau187P301L aggregation, we report on the evolution of the relative areas of each component during the amyloid formation (figure 6). This relative area provides information about the ratio of events detected for each population. Note that this parameter is related, but not directly proportional to the concentration of each population. Indeed, to correlate the area with the concentration, several parameters have to be taken into account, such as the diffusion coefficient of the aggregates and the energy barrier of entrance into the detection zone ${ }^{59,60}$. However, the relative area can still provide meaningful qualitative information. The populations at 70 and $80 \mathrm{~min}$ are not represented because it is not possible to discriminate the monomer from the oligomer populations (see figure 4), and thus we cannot plot their respective rate areas.

For tau187WT, the population of oligomers after 120 min represents $57 \%$, while the remaining $43 \%$ are monomers (figure 6a). The fibrils population observed at $198 \mathrm{~min}$ and $256 \mathrm{~min}$ are low (7\% and 5\% respectively). This population of fibrils disappears at $372 \mathrm{~min}$, letting only oligomers detectable. For tau187P301L, the population of oligomers increases at low time and quickly converts to higher order assemblies. At $138 \mathrm{~min}$, the momomer population is completely transformed into oligomers (43\%) and fibrils (57\%). Note that these two populations did not reach their final size according to their intermediate values of $\Delta l / I_{0}$ (see figure $5 \mathrm{~b}$ ). From 138 min to $200 \mathrm{~min}$, the population of fibrils drastically reduces from $53 \%$ to $2 \%$, before slightly increasing to $11 \%$ at longer time.

These population variations can be interpreted in the following way. At short times ( 130 min), most of the species have not overgrown the size of the nanopore (see previous discussion) and are therefore detected. The faster conversion rate to oligomer found for P301L compared to WT (figure 5) are reflected by the complete disappearance of P301L. At $\sim 130 \mathrm{~min}$, for tau187P301L we detected $43 \%$ of oligomers and $57 \%$ or fibrils while the tau187WT samples was composed of $\sim 43 \%$ monomer and $57 \%$ oligomers. At intermediate times, fibrils overgrow the nanopore size and therefore give rise to a diminution of the apparent fibril population (from $57 \%$ to $2 \%$ between 130 and 200 min for P301L). At longer time, although mature fibrils are no longer detected, broken fibrils can significantly contribute to the observed population of larger aggregate population. Because fibrils breakage is more prominent for $\mathrm{P} 301 \mathrm{~L}^{49}$, the populations of broken fibrils contribute to the increase of fibrils (from $2 \%$ to $200 \mathrm{~min}$ to $11 \%$ at $350 \mathrm{~min}$ ) population with the time, while it decreases for the WT. 

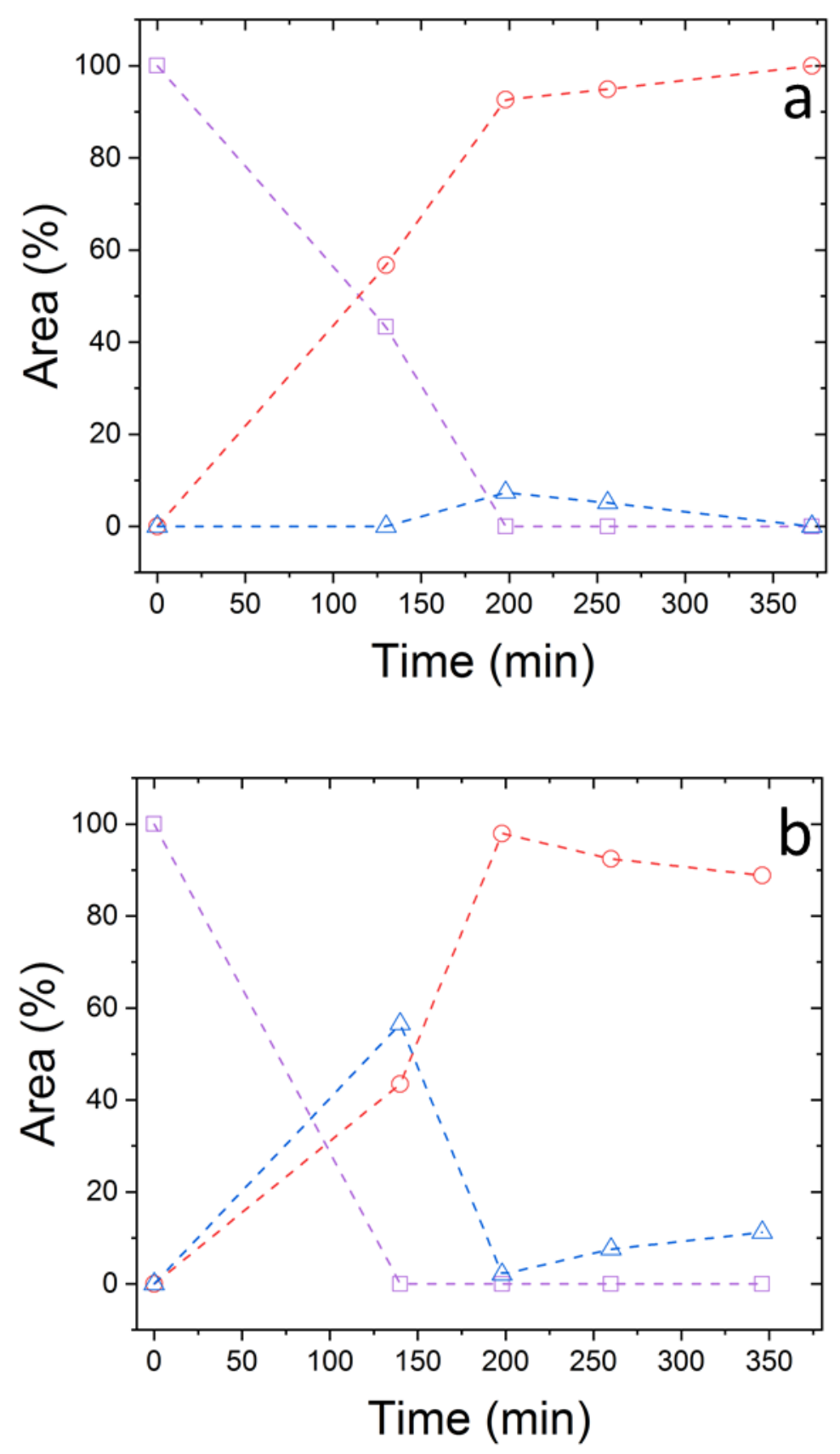

Figure 6. Analysis of the area determined for the data in figure 3. The ratio of monomers (violet, square) oligomer (red, circle), fibrils (blue, triangle) are reported as a function of the time for tau187WT (a) and tau187P301L(b).

\section{Comparison of long event structures between tau187WT and tau187P301L}

The nanopore sensing can also provide information about the structure or conformation of the detected objects. Indeed, the current blockade structure can be assigned to a fingerprint, as previously shown for other proteins including streptavidin, monoclonal and polyclonal antibody and $A \beta^{26,31}$. In Figure $1 c$, the amplitude of relative current blockade obtained before heparin addition could be associated to a monomer. We can estimate its hydrodynamic radius at about $3 \mathrm{~nm}$ using the model previously proposed by Giamblanco et $\mathrm{al}^{38}$, in good agreement with the hydrodynamic radius of tau K18 (244-372) measured by dynamic light scattering ${ }^{61}$. 

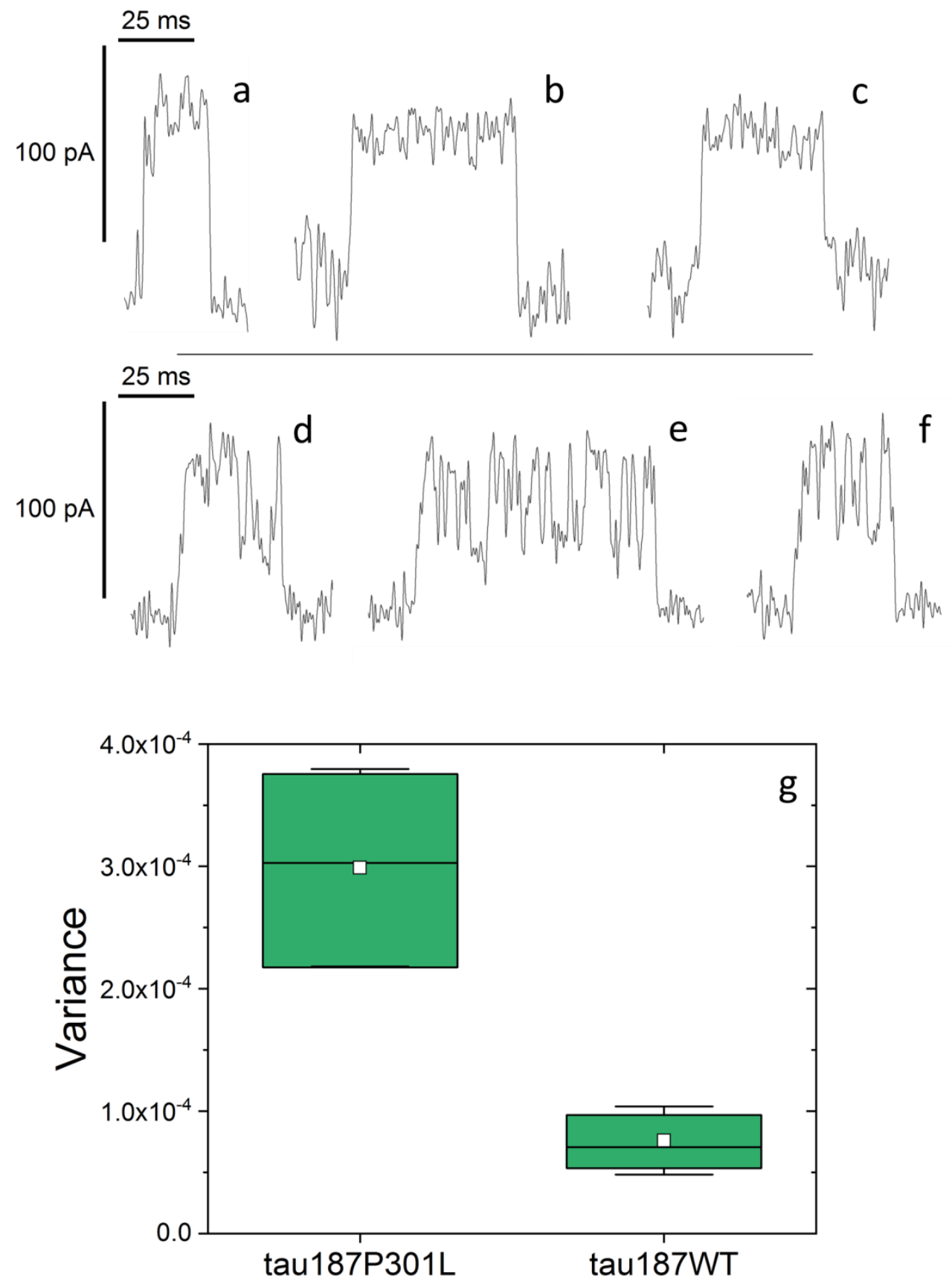

Figure 7. Examples of medium current blockades $(100 \mathrm{~ms}$ to $1 \mathrm{sec})$ recorded at $-200 \mathrm{mV}$ after various incubation times for tau187WT $(a, b, c)$ tau187P301L (d,e,f). g Box plots displaying the variance of current blockade signal, the green boxes represents the quartile 25\%$75 \%$, the mean value (white, square), the median (black line) and whiskers are the standard deviation over $\mathrm{N}=45$. 

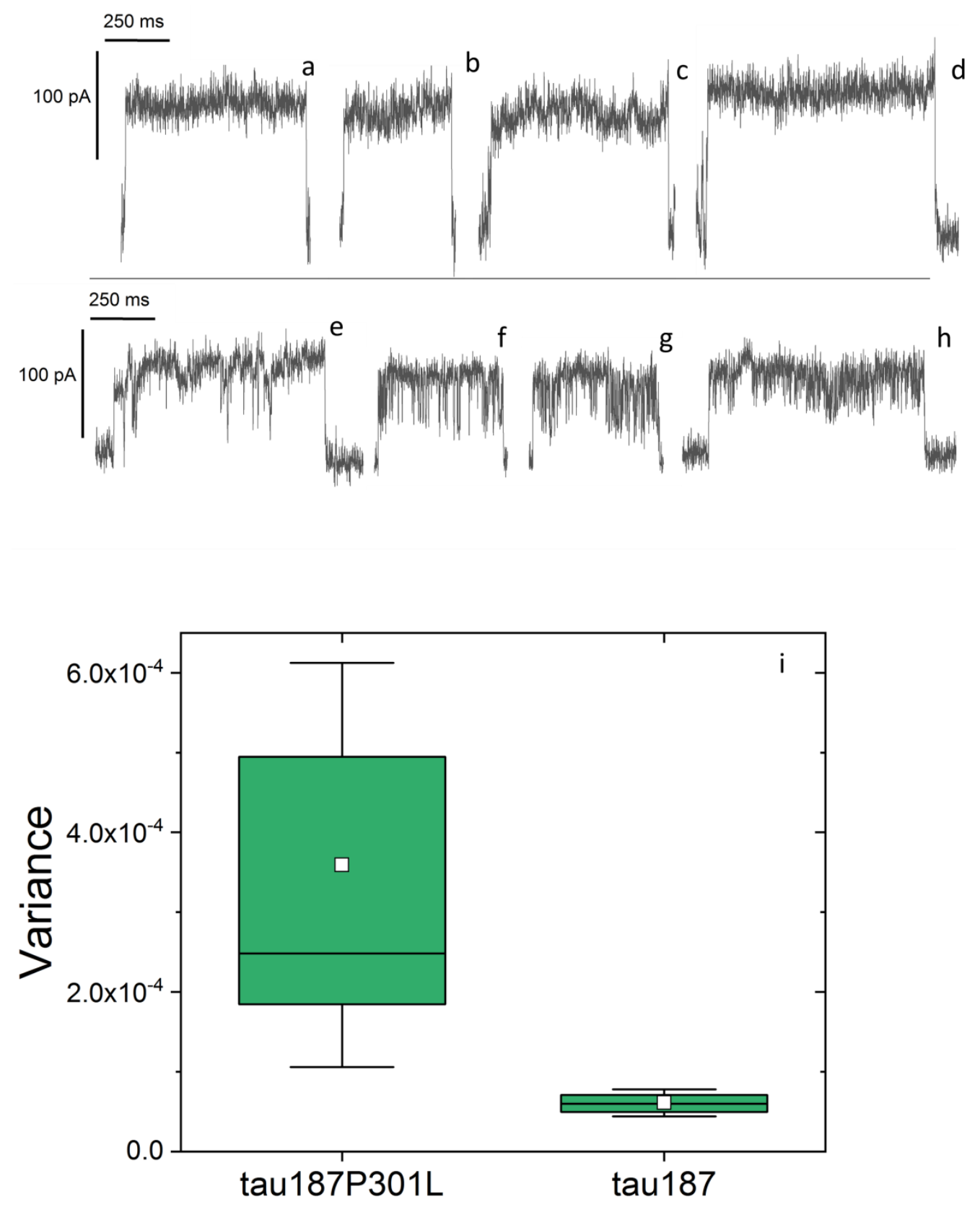

Figure 8. Examples of long current blockades (greater than $1 \mathrm{sec}$ ) recorded at $-200 \mathrm{mV}$ after various incubation times for tau187WT $(a, b, c, d)$ tau187P301L (e,f,g,h). (i) Box plots displaying the variance of current blockade signal, the green boxes represents the quartile $25 \%-75 \%$, the mean value (white, square), the median (black line) and whiskers are the standard deviation over $N=45$.

Translocation of amyloid through the nanopore produces typical resistive pulse reported in figure $7,8, \mathrm{SI}-5$ to SI8. The amplitude and the duration are related to the size of the protein aggregates. However, a deeper analysis of the current blockade can reveal other interesting information. For example, Mayer's group has reported that the current fluctuation in the case of spheroid proteins anchoring to a wall lipid bilayer evidences their rotation ${ }^{26}$. Here, the details of the blockage signal reflect the conformational stability within the aggregates confined in the sensing volume. In our experiment, the medium (between $100 \mathrm{~ms}$ and $1 \mathrm{~s}$ ) and long events (several seconds) contain two interesting information (figures 7-8 and SI-5-SI-8). First, for the medium events, we observe larger current fluctuations (greater than $40 \mathrm{pA}$ ) for tau187P301L (figure 7d-f and Si-6) than for tau187WT (figure 7a-c 
and SI-5). This is confirmed by the larger variance of the blockade signal obtained for tau187P301L (mean value = $2.9910^{-4}$ ) than tau187WT (mean value $=0.7610^{-4}$ ) (Figure $7 \mathrm{~g}$ ). Because amyloid structure are very stables, conformational fluctuations mostly occurred in the part of the protein that remain unstructured in the fibrils, socalled fuzzy coat ${ }^{62}$, which is expected to be composed of the $100 \mathrm{c}$-terminal amino acids of tau $187^{14}$. Although more in depth measurements are necessary to obtain a precise structural picture, our results suggest that tau187P301L fiber possess a more stiff/restrain fuzzy coat than tau187WT fibers. For the long events (several seconds) additionally to the fluctuations, we observe for the tau187P301L many spikes relative to the current enhancement (Figure 8e-h and SI-8) due to a quick increase of the concentration of mobile ion inside the detection zone. Conversely the long events recorded for tau187WT (figures 8a-d and SI-7) did not exhibit such spikes. The current spikes might reflect fibril breakage occurring in the sensing regions, supporting our interpretation of the populations (figure 4) that fibril breakage is much more prominent for tau187P301L than tau187WT.

\section{Conclusions}

To sum up, we used conical nanopores to characterize heparin-induced aggregation of a tau construct, tau187, with and without P301L mutation. From the relative current blockade and the dwell time, we have differentiated three populations, which we assigned to monomers, oligomers and fibrils. The kinetic analysis of aggregation growth of each population allows to extract growth rates and highlights that the tau187P301L is more prone to form oligomer as compared to tau187WT. Indeed, the total transformation of monomer is faster for the tau187P301L than tau187WT. A fragmentation mechanism for tau187P301L fibrils is also reported both from the population analysis and the current enhancement observed in the current blockade. Furthermore, we suggest that the fluctuation observed in the blockade current for tau187P301L can be assigned to a structural flexibility higher in tau187P301L than in tau187WT aggregates. Together these results confirm that the conical nanopore is a powerful tool to characterize amyloid growth and improve our understanding of amyloid formation, fragmentation and accumulation.

\section{ASSOCIATED CONTENT}

Supporting Information Available: The following files are available free of charge. Characterisation of nanopore, scatter plot of translocation events for tau187WT and tau187P301L before and after heparin addition, capture rate of tau187WT and tau187P301L a function of incubation time with heparin and additional examples of current blockades.

\section{Acknowledgements}

SB acknowledges the co-funded by Région Languedoc-Roussillon and European Union (FEDER) - grant "chercheur d'avenir - NanoDiag". SB and NG acknowledge LabEX Chemisyst (ANR- 10-LABX605-UI) - Université de Montpellier project "Amydiag". YF and SH acknowledge the Tau Consortium (https://tauconsortium.org/) from the Rainwater Foundation and NIH Grant R01AG056058. We acknowledge the use of the NRI-MCDB Microscopy Facility at UC, Santa Barbara. Single tracks have been produced in GANIL (Caen, France) in the framework of an EMIR project.

\section{Notes and references}


(1) Lee, V. M.-Y.; Goedert, M.; Trojanowski, J. Q. Neurodegenerative Tauopathies. Annu. Rev. Neurosci. 2001, 24 (1), 1121-1159. https://doi.org/10.1146/annurev.neuro.24.1.1121.

(2) Lasagna-Reeves, C. A.; Castillo-Carranza, D. L.; Sengupta, U.; Sarmiento, J.; Troncoso, J.; Jackson, G. R.; Kayed, R. Identification of Oligomers at Early Stages of Tau Aggregation in Alzheimer's Disease. FASEB J. 2012, 26 (5), 1946-1959. https://doi.org/10.1096/fj.11-199851.

(3) Patterson, K. R.; Remmers, C.; Fu, Y.; Brooker, S.; Kanaan, N. M.; Vana, L.; Ward, S.; Reyes, J. F.; Philibert, K.; Glucksman, M. J.; et al. Characterization of Prefibrillar Tau Oligomers in Vitro and in Alzheimer Disease. J. Biol. Chem. 2011, 286 (26), 23063-23076. https://doi.org/10.1074/jbc.M111.237974.

(4) Maeda, S.; Sahara, N.; Saito, Y.; Murayama, S.; Ikai, A.; Takashima, A. Increased Levels of Granular Tau Oligomers: An Early Sign of Brain Aging and Alzheimer's Disease. Neurosci. Res. 2006, 53 (3), 197-201. https://doi.org/10.1016/j.neures.2005.11.009.

(5) Shafiei, S. S.; Guerrero-Muñoz, M. J.; Castillo-Carranza, D. L. Tau Oligomers: Cytotoxicity, Propagation, and Mitochondrial Damage. Front. Aging Neurosci. 2017, 9 (APR), 1-9. https://doi.org/10.3389/fnagi.2017.00083.

(6) Weingarten, M. D.; Lockwood, A. H.; Hwo, S.; Kirschner, M. W. A Protein Factor Essential for Microtubule Assembly. Proc. Natl. Acad. Sci. 1975, 72 (5), 1858-1862.

(7) Brandt, R.; Lee, G. Functional Organization of Microtubule-Associated Protein Tau. J. Biol. Chem. 1993, 268 (5), 3414-3419.

(8) Fitzpatrick, A. W. P.; Falcon, B.; He, S.; Murzin, A. G.; Murshudov, G.; Garringer, H. J.; Crowther, R. A.; Ghetti, B.; Goedert, M.; Scheres, S. H. W. Cryo-EM Structures of Tau Filaments from Alzheimer's Disease. Nature 2017, 547, 185-190. https://doi.org/10.1038/nature23002.

(9) Falcon, B.; Zhang, W.; Murzin, A. G.; Murshudov, G.; Garringer, H. J.; Vidal, R.; Crowther, R. A.; Ghetti, B.; Scheres, S. H. W.; Goedert, M. Structures of Filaments from Pick's Disease Reveal a Novel Tau Protein Fold. Nature 2018, 561, 137. https://doi.org/10.1038/s41586-018-0454-y.

(10) Falcon, B.; Zivanov, J.; Zhang, W.; Murzin, A. G.; Garringer, H. J.; Vidal, R.; Crowther, R. A.; Newell, K. L.; Ghetti, B.; Goedert, M.; et al. Novel Tau Filament Fold in Chronic Traumatic Encephalopathy Encloses Hydrophobic Molecules. Nature 2019, 568, 420-423. https://doi.org/10.1038/s41586-019-1026-5.

(11) Goedert, M.; Jakes, R.; Spillantini, M. G.; Hasegawa, M.; Smith, M. J.; Crowther, R. A. Assembly of Microtubule-Associated Protein Tau into Alzheimer-like Filaments Induced by Sulphated Glycosaminoglycans. Nature 1996, 383, 550-553. https://doi.org/10.1038/383550a0.

(12) Kampers, T.; Friedhoff, P.; Biernat, J.; Mandelkow, E. M.; Mandelkow, E. RNA Stimulates Aggregation of Microtubule-Associated Protein Tau into Alzheimer-like Paired Helical Filaments. FEBS Lett. 1996, 399, 344-349. https://doi.org/10.1016/S0014-5793(96)01386-5.

(13) Wilson, D. M.; Binder, L. I. Free Fatty Acids Stimulate the Polymerization of Tau and Amyloid Beta Peptides. In Vitro Evidence for a Common Effector of Pathogenesis in Alzheimer's Disease. Am. J. Pathol. 1997, 150, 2181-2195. https://doi.org/10.1073/pnas.85.12.4506.

(14) Zhang, W.; Falcon, B.; Murzin, A. G.; Fan, J.; Crowther, R. A.; Goedert, M.; Scheres, S. H. Heparin-Induced Tau Filaments Are Polymorphic and Differ from Those in Alzheimer's and Pick's Diseases. Elife 2019, 8, e43584. https://doi.org/10.7554/elife.43584.

(15) Fichou, Y.; Vigers, M.; Goring, A. K.; Eschmann, N. A.; Han, S. Heparin-Induced Tau Filaments Are Structurally Heterogeneous and Differ from Alzheimer's Disease Filaments. Chem. Commun. 2018, 54, 
4573-4576. https://doi.org/10.1039/c8cc01355a.

(16) Pavlova, A.; Cheng, C.-Y.; Kinnebrew, M.; Lew, J.; Dahlquist, F. W.; Han, S. Protein Structural and Surface Water Rearrangement Constitute Major Events in the Earliest Aggregation Stages of Tau. Proc. Natl. Acad. Sci. 2016, 113 (2), E127-E136. https://doi.org/10.1073/pnas.1504415113.

(17) Lasagna-Reeves, C. A.; Castillo-Carranza, D. L.; Sengupta, U.; Sarmiento, J.; Troncoso, J.; Jackson, G. R.; Kayed, R. Identification of Oligomers at Early Stages of Tau Aggregation in Alzheimer's Disease. FASEB J. 2012, 26 (5), 1946-1959. https://doi.org/10.1096/fj.11-199851.

(18) Shammas, S. L.; Garcia, G. A.; Kumar, S.; Kjaergaard, M.; Horrocks, M. H.; Shivji, N.; Mandelkow, E.; Knowles, T. P. J.; Mandelkow, E.; Klenerman, D. A Mechanistic Model of Tau Amyloid Aggregation Based on Direct Observation of Oligomers. Nat. Commun. 2015, 6, 7025. https://doi.org/10.1038/ncomms8025.

(19) Wang, Y.; Mandelkow, E. Tau in Physiology and Pathology. Nat. Rev. Neurosci. 2016, 17 (1), 5-21. https://doi.org/10.1038/nrn.2015.1.

(20) Meyer, V.; Dinkel, P. D.; Luo, Y.; Yu, X.; Wei, G.; Zheng, J.; Eaton, G. R.; Ma, B.; Nussinov, R.; Eaton, S. S.; et al. Single Mutations in Tau Modulate the Populations of Fibril Conformers through Seed Selection. Angew. Chemie - Int. Ed. 2014, 53 (6), 1590-1593. https://doi.org/10.1002/anie.201308473.

(21) Nilsson, M. R. Techniques to Study Amyloid Fibril Formation in Vitro. Methods 2004, 34 (1), 151-160. https://doi.org/10.1016/j.ymeth.2004.03.012.

(22) Chaari, A.; Fahy, C.; Chevillot-Biraud, A.; Rholam, M. Insights into Kinetics of Agitation-Induced Aggregation of Hen Lysozyme under Heat and Acidic Conditions from Various Spectroscopic Methods. PLoS One 2015, 10 (11). https://doi.org/10.1371/journal.pone.0142095.

(23) Langkilde, A. E.; Vestergaard, B. Methods for Structural Characterization of Prefibrillar Intermediates and Amyloid Fibrils. FEBS Lett. 2009, 583 (16), 2600-2609. https://doi.org/10.1016/j.febslet.2009.05.040.

(24) Houghtaling, J.; List, J.; Mayer, M. Nanopore-Based, Rapid Characterization of Individual Amyloid Particles in Solution: Concepts, Challenges, and Prospects. Small 2018, 1802412.

https://doi.org/10.1002/smll.201802412.

(25) Kasianowicz, J. J.; Brandin, E.; Branton, D.; Deamer, D. W. Characterization of Individual Polynucleotide Molecules Using a Membrane Channel. Proc. Natl. Acad. Sci. U. S. A. 1996, 93 (24), 13770-13773. https://doi.org/10.1073/pnas.93.24.13770.

(26) Yusko, E. C.; Bruhn, B. R.; Eggenberger, O. M.; Houghtaling, J.; Rollings, R. C.; Walsh, N. C.; Nandivada, S.; Pindrus, M.; Hall, A. R.; Sept, D.; et al. Real-Time Shape Approximation and Fingerprinting of Single Proteins Using a Nanopore. Nat. Nanotechnol. 2016, 12 (4), 360-367.

https://doi.org/10.1038/nnano.2016.267.

(27) Piguet, F.; Ouldali, H.; Pastoriza-Gallego, M.; Manivet, P.; Pelta, J.; Oukhaled, A. Identification of Single Amino Acid Differences in Uniformly Charged Homopolymeric Peptides with Aerolysin Nanopore. Nat. Commun. 2018, 9, 966. https://doi.org/10.1038/s41467-018-03418-2.

(28) Wang, H. Y.; Ying, Y. L.; Li, Y.; Kraatz, H. B.; Long, Y. T. Nanopore Analysis of $\beta$-Amyloid Peptide Aggregation Transition Induced by Small Molecules. Anal. Chem. 2011, 83 (5), 1746-1752.

https://doi.org/10.1021/ac1029874.

(29) Zhao, Q.; De Zoysa, R. S. S.; Wang, D.; Jayawardhana, D. A.; Guan, X. Real-Time Monitoring of Peptide Cleavage Using a Nanopore Probe. J. Am. Chem. Soc. 2009, 131 (18), 6324-6325.

https://doi.org/10.1021/ja9004893. 
(30) Balme, S.; Coulon, P. E.; Lepoitevin, M.; Charlot, B.; Yandrapalli, N.; Favard, C.; Muriaux, D.; Bechelany, M.; Janot, J. M. Influence of Adsorption on Proteins and Amyloid Detection by Silicon Nitride Nanopore. Langmuir 2016, 32 (35), 8916-8925. https://doi.org/10.1021/acs.langmuir.6b02048.

(31) Yusko, E. C.; Johnson, J. M.; Majd, S.; Prangkio, P.; Rollings, R. C.; Li, J.; Yang, J.; Mayer, M. Controlling Protein Translocation through Nanopores with Bio-Inspired Fluid Walls. Nat. Nanotechnol. 2011, 6 (4), 253-260. https://doi.org/10.1038/nnano.2011.12.

(32) Yusko, E. C.; Prangkio, P.; Sept, D.; Rollings, R. C.; Li, J.; Mayer, M. Single-Particle Characterization of A $\beta$ Oligomers in Solution. ACS Nano 2012, 6 (7), 5909-5919. https://doi.org/10.1021/nn300542q.

(33) Hu, R.; Diao, J.; Li, J.; Tang, Z.; Li, X.; Leitz, J.; Long, J.; Liu, J.; Yu, D.; Zhao, Q. Intrinsic and MembraneFacilitated $\alpha$-Synuclein Oligomerization Revealed by Label-Free Detection through Solid-State Nanopores. Sci. Rep. 2016, 6 (January), 20776. https://doi.org/10.1038/srep20776.

(34) Giamblanco, N.; Coglitore, D.; Janot, J.; Eugène, P.; Charlot, B.; Balme, S. Sensors and Actuators B : Chemical Detection of Protein Aggregate Morphology through Single Antifouling Nanopore. Sensors Actuators B. Chem. 2018, 260, 736-745. https://doi.org/10.1016/j.snb.2018.01.094.

(35) Li, W.; Bell, N. A. W.; Hernández-Ainsa, S.; Thacker, V. V.; Thackray, A. M.; Bujdoso, R.; Keyser, U. F. Single Protein Molecule Detection by Glass Nanopores. ACS Nano 2013, 7 (5), 4129-4134. https://doi.org/10.1021/nn4004567.

(36) Martyushenko, N.; Bell, N. A. W.; Lamboll, R. D.; Keyser, U. F. Nanopore Analysis of Amyloid Fibrils Formed by Lysozyme Aggregation. Analyst 2015, 140, 4882-4886. https://doi.org/10.1039/c5an00530b.

(37) Yu, R.-J.; Lu, S.-M.; Xu, S.-W.; Li, Y.-J.; Xu, Q.; Ying, Y.-L.; Long, Y.-T. Single Molecule Sensing of Amyloid- $\beta$ Aggregation by Confined Glass Nanopore. Chem. Sci. 2019. https://doi.org/10.1039/c9sc03260f.

(38) Giamblanco, N.; Coglitore, D.; Gubbiotti, A.; Ma, T.; Balanzat, E.; Janot, J. M.; Chinappi, M.; Balme, S. Amyloid Growth, Inhibition, and Real-Time Enzymatic Degradation Revealed with Single Conical Nanopore. Anal. Chem. 2018, 90, 12900-12908. https://doi.org/10.1021/acs.analchem.8b03523.

(39) Hutton, M.; Froelich, S.; Houlden, H.; Pickering-brown, S.; Chakraverty, S.; Isaacs, A.; Grover, A.; Hackett, J.; Adamson, J.; Lincoln, S.; et al. Association of Missense and 5'-Splice-Site Mutations in Tau with the Inherited Dementia FTDP-17. Nature 1998, 393 (June), 702-705.

(40) van Oss, C. J. A Review of: "Particle Size Analysis", N.G. Stanley-Wood and R.W. Lines, Eds., Royal Society of Chemistry, Cambridge, 1992; CRC Press/Lewis Publishers, Boca Raton, FL; Pp. Xix + 538, \$119. . J. Dispers. Sci. Technol. 2007, 14 (5), 606-606. https://doi.org/10.1080/01932699308943432.

(41) Fichou, Y.; Lin, Y.; Rauch, J. N.; Vigers, M.; Zeng, Z.; Srivastava, M.; Keller, T. J.; Freed, J. H.; Kosik, K. S.; Han, S. Cofactors Are Essential Constituents of Stable and Seeding-Active Tau Fibrils. Proc. Natl. Acad. Sci. U. S. A. 2018, 115, 13234-13239. https://doi.org/10.1073/pnas.1810058115.

(42) Lepoitevin, M.; Ma, T.; Bechelany, M.; Janot, J.-M.; Balme, S. Functionalization of Single Solid State Nanopores to Mimic Biological Ion Channels: A Review. Adv. Colloid Interface Sci. 2017, 250, 195-213. https://doi.org/10.1016/j.cis.2017.09.001.

(43) Lepoitevin, M.; Jamilloux, B.; Bechelany, M.; Balanzat, E.; Janot, J.-M.; Balme, S. Fast and Reversible Functionalization of a Single Nanopore Based on Layer-by-Layer Polyelectrolyte Self-Assembly for Tuning Current Rectification and Designing Sensors. RSC Adv. 2016, 6 (38), 32228-32233. https://doi.org/10.1039/c6ra03698h.

(44) Zhao, J.; Huvent, I.; Lippens, G.; Eliezer, D.; Zhang, A.; Li, Q.; Tessier, P.; Linhardt, R. J.; Zhang, F.; Wang, C. 
Glycan Determinants of Heparin-Tau Interaction. Biophys. J. 2017, 112 (5), 921-932. https://doi.org/10.1016/j.bpj.2017.01.024.

(45) Roman, J.; Jarroux, N.; Patriarche, G.; Français, O.; Pelta, J.; Le Pioufle, B.; Bacri, L. Functionalized SolidState Nanopore Integrated in a Reusable Microfluidic Device for a Better Stability and Nanoparticle Detection. ACS Appl. Mater. Interfaces 2017, 9, 41634-41640. https://doi.org/10.1021/acsami.7b14717.

(46) Baaken, G.; Halimeh, I.; Bacri, L.; Pelta, J.; Oukhaled, A.; Behrends, J. C. High-Resolution Size-Discrimination of Single Nonionic Synthetic Polymers with a Highly Charged Biological Nanopore. ACS Nano 2015, 9 (6), 6443-6449. https://doi.org/10.1021/acsnano.5b02096.

(47) Kjaergaard, M.; Dear, A. J.; Kundel, F.; Qamar, S.; Meisl, G.; Knowles, T. P. J.; Klenerman, D. Oligomer Diversity during the Aggregation of the Repeat-Region of Tau. ACS Chem. Neurosci. 2018, 19, 3060-3071. https://doi.org/10.1021/acschemneuro.8b00250.

(48) Ramachandran, G.; Udgaonkar, J. B. Understanding the Kinetic Roles of the Inducer Heparin and of Rodlike Protofibrils during Amyloid Fibril Formation by Tau Protein. J. Biol. Chem. 2011, 286 (45), 3894838959. https://doi.org/10.1074/jbc.M111.271874.

(49) Kundel, F.; Hong, L.; Falcon, B.; McEwan, W. A.; Michaels, T. C. T.; Meisl, G.; Esteras, N.; Abramov, A. Y.; Knowles, T. J. P.; Goedert, M.; et al. Measurement of Tau Filament Fragmentation Provides Insights into Prion-like Spreading. ACS Chem. Neurosci. 2018, 9 (6), 1276-1282. https://doi.org/10.1021/acschemneuro.8b00094.

(50) Larkin, J.; Henley, R. Y.; Muthukumar, M.; Rosenstein, J. K.; Wanunu, M. High-Bandwidth Protein Analysis Using Solid-State Nanopores. Biophys. J. 2014, 106 (3), 696-704.

https://doi.org/10.1016/j.bpj.2013.12.025.

(51) Plesa, C.; Kowalczyk, S. W.; Zinsmeester, R.; Grosberg, A. Y.; Rabin, Y.; Dekker, C. Fast Translocation of Proteins through Solid State Nanopores. Nano Lett. 2013, 13 (2), 658-663. https://doi.org/10.1021/nl3042678.

(52) Hong, L.; Qi, X.; Zhang, Y. Dissecting the Kinetic Process of Amyloid Fiber Formation through Asymptotic Analysis. J. Phys. Chem. B 2012, 116 (23), 6611-6617. https://doi.org/10.1021/jp205702u.

(53) Linse, S. Monomer-Dependent Secondary Nucleation in Amyloid Formation. Biophys. Rev. 2017, 9 (4), 329 338. https://doi.org/10.1007/s12551-017-0289-z.

(54) Nakatani-Webster, E.; Nath, A. Inferring Mechanistic Parameters from Amyloid Formation Kinetics by Approximate Bayesian Computation. Biophys. J. 2017, 112 (5), 868-880.

https://doi.org/10.1016/j.bpj.2017.01.011.

(55) Eden, K.; Morris, R.; Gillam, J.; Macphee, C. E.; Allen, R. J. Competition between Primary Nucleation and Autocatalysis in Amyloid Fibril Self-Assembly. Biophys. J. 2015, 108 (3), 632-643. https://doi.org/10.1016/j.bpj.2014.11.3465.

(56) Bhabha, G.; Lee, J.; Ekiert, D. C.; Gam, J.; Wilson, I. A.; Jane, H.; Benkovic, S. J.; Wright, P. E. Influence the Chemical Step of Enzyme Catalysis. October 2011, 332 (6026), 234-238.

https://doi.org/10.1126/science.1198542.A.

(57) Knowles, T.; Waudby, C.; Devlin, G.; Cohen, S.; Aguzzi, A.; Vendruscolo, M.; Terentjev, E.; Welland, M.; Dobson, C. An Analytical Solution to the Kinetics of Breakable Filament Assembly. Science (80-. ). 2009, 326, 1533-1537.

(58) Zhu, H. L.; Fernández, C.; Fan, J. B.; Shewmaker, F.; Chen, J.; Minton, A. P.; Liang, Y. Quantitative 
Characterization of Heparin Binding to Tau Protein: Implication for Inducer-Mediated Tau Filament Formation. J. Biol. Chem. 2010, 285 (6), 3592-3599. https://doi.org/10.1074/jbc.M109.035691.

(59) Cabello-Aguilar, S.; Chaaya, A. A.; Bechelany, M.; Pochat-Bohatier, C.; Balanzat, E.; Janot, J.-M.; Miele, P.; Balme, S. Dynamics of Polymer Nanoparticles through a Single Artificial Nanopore with a High-AspectRatio. Soft Matter 2014, 10 (42), 8413-8419. https://doi.org/10.1039/c4sm00392f.

(60) Bacri, L.; Oukhaled, A. G.; Schiedt, B.; Patriarche, G.; Bourhis, E.; Gierak, J.; Pelta, J.; Auvray, L. Dynamics of Colloids in Single Solid-State Nanopores. J. Phys. Chem. B 2011, 115 (12), 2890-2898. https://doi.org/10.1021/jp200326w.

(61) Akoury, E.; Mukrasch, M. D.; Biernat, J.; Tepper, K.; Ozenne, V.; Mandelkow, E.; Blackledge, M.; Zweckstetter, M. Remodeling of the Conformational Ensemble of the Repeat Domain of Tau by an Aggregation Enhancer. Protein Sci. 2016, 25, 1010-1020. https://doi.org/10.1002/pro.2911.

(62) Wegmann, S.; Medalsy, I. D.; Mandelkow, E.; Müller, D. J. The Fuzzy Coat of Pathological Human Tau Fibrils Is a Two-Layered Polyelectrolyte Brush. Proc. Natl. Acad. Sci. U. S. A. 2012, 110 (4), E313-E321. https://doi.org/10.1073/pnas.1212100110. 